\title{
MyD88-dependent induction of allergic Th2 responses to intranasal antigen
}

\author{
Damani A. Piggott, ${ }^{1}$ Stephanie C. Eisenbarth, ${ }^{1}$ Lan Xu, ${ }^{1}$ Stephanie L. Constant, ${ }^{2}$ \\ James W. Huleatt, ${ }^{1}$ Christina A. Herrick, ${ }^{1,3}$ and Kim Bottomly1,3
}

\begin{abstract}
${ }^{1}$ Section of Immunobiology, Yale University School of Medicine, New Haven, Connecticut, USA. ${ }^{2}$ Department of Microbiology and Tropical Medicine, George Washington University, Washington, DC, USA. ${ }^{3}$ Department of Dermatology, Yale University School of Medicine, New Haven, Connecticut, USA.
\end{abstract}

\begin{abstract}
MyD88 is a common Toll-like receptor (TLR) adaptor molecule found to be essential for induction of adaptive Th1 immunity. Conversely, innate control of adaptive Th2 immunity has been shown to occur in a MyD88independent manner. In this study, we show that MyD88 is an essential innate component in the induction of TLR4-dependent Th2 responses to intranasal antigen; thus we demonstrate what we believe to be a novel role for MyD88 in pulmonary Th2 immunity. Induction of the MyD88-independent type I IFN response to LPS is defective in the pulmonary environment. Moreover, in the absence of MyD88, LPS-induced upregulation of costimulatory molecule expression on pulmonary DCs is defective, in contrast to what has been observed with bone marrow-derived DCs (BMDCs). Reconstitution of Th2 responses occurs upon adoptive pulmonary transfer of activated BMDCs to MyD88-deficient recipients. Furthermore, the dependence of Th2 responses on MyD88 is governed by the initial route of antigen exposure; this demonstrates what we believe are novel site-specific innate mechanisms for control of adaptive Th2 immunity.
\end{abstract}

\section{Introduction}

The immune system is constantly faced with the challenge of generating a potent defense against pathogenic organisms while maintaining tolerance to both self and innocuous environmental antigens. Appropriate control of adaptive immune responses is a central element in preserving this fine balance. When this balance is lost, pathological conditions such as autoimmunity and impaired resistance to infection ensue. Toll-like receptors (TLRs) have been found to be critical players in appropriate and balanced immune responses.

Lymphocyte activation is highly regulated. In particular, CD4 ${ }^{+}$ $T$ cell activation is dependent on the interaction of the TCR with MHC class II:peptide complexes on APCs $(1,2)$. In addition, full responsiveness requires the engagement of $\mathrm{CD} 28$ by costimulatory molecules such as CD80 or CD86 $(3,4)$. Innate immune system signals, such as those delivered through TLRs, are believed to be pivotal to the regulation of both MHC class II:peptide and costimulatory molecule expression, allowing the host to discriminate self from infectious non-self. Induction of inflammatory cytokines including TNF- $\alpha$ and type I IFN by TLRs further ensures potent responses to infectious agents (5-7).

MyD88 is a critical common adaptor molecule shared by TLRs $(8,9)$. Signaling through several TLRs is completely MyD88 dependent. However, MyD88-independent pathways exist for some TLRs $(10,11)$. Prior studies have demonstrated that in the absence of MyD88, Th1 responses are abrogated, which indicates a clear role for TLR/MyD88-dependent signaling in the control of adaptive Th1 immunity. Conversely, studies have demonstrated intact or augmented Th2 responses in the absence of MyD88 (12-16). These

Nonstandard abbreviations used: BAL, bronchoalveolar lavage; BMDC, bone marrow-derived DC; OVA/LPS, OVA with low-dose LPS; TLR, Toll-like receptor; TLR4d, C.C3H-TLR4 ${ }^{\text {LPS-d; }}$ TRIF, TIR domain-containing adaptor inducing IFN- $\beta$.

Conflict of interest: The authors have declared that no conflict of interest exists.

Citation for this article: J. Clin. Invest. 115:459-467 (2005).

doi:10.1172/JCI200522462. data suggested that the elicitation of Th2 responses involves innate immune signals that are MyD88 independent, and therefore TLR independent. However, given the ability of several TLRs to signal in a MyD88-independent manner, it remained conceivable that Th2 responses could be elicited through a TLR-dependent/MyD88independent pathway. Support for the latter hypothesis came from a study demonstrating that bone marrow-derived DCs (BMDCs) lacking MyD88 can induce Th2 responses to LPS in vitro through the engagement of a TLR4-dependent signaling pathway (12). This study thus demonstrated a TLR4-dependent/MyD88-independent mechanism for Th2 generation.

In further support of the existence of a TLR-dependent mode of Th2 generation, our and others' recent in vivo findings indicate that Th2 responses to OVA are TLR4 dependent $(17,18)$. Using a murine model of asthma, characterized by airway inflammation, eosinophilia, and mucus hypersecretion in response to intranasal antigen, we found that the dose of LPS regulates the induction of Th2 versus Th1 responses, with intranasal antigen requiring concomitant low-dose LPS signaling through TLR4 to induce allergic pulmonary Th2 responses. LPS signaling through TLR4 engages 2 major pathways of immune activation. Production of inflammatory cytokines such as TNF- $\alpha$ has been demonstrated to be MyD88 dependent. Conversely, LPS/TLR4-responsive upregulation of costimulatory molecules, and the induction of IFN- $\beta$ and a cassette of IFN-responsive immune mediators, have been demonstrated previously to be MyD88 independent (19-22). Since previous studies supported the concept that Th2 responses remain intact in the absence of MyD88, and given that LPS signaling through TLR4 engages both MyD88dependent and MyD88-independent pathways (20, 21, 23, 24), we questioned whether the TLR4-dependent generation of allergic Th2 pulmonary responses resulted from LPS engagement of the TLR4dependent/MyD88-independent pathway.

We show in the present study that Th2 sensitization to intranasal antigen in the presence of LPS is MyD88 dependent, in part because of the requirement of MyD88 for effective pulmonary DC maturation in response to LPS. Such MyD88-dependent maturation of 

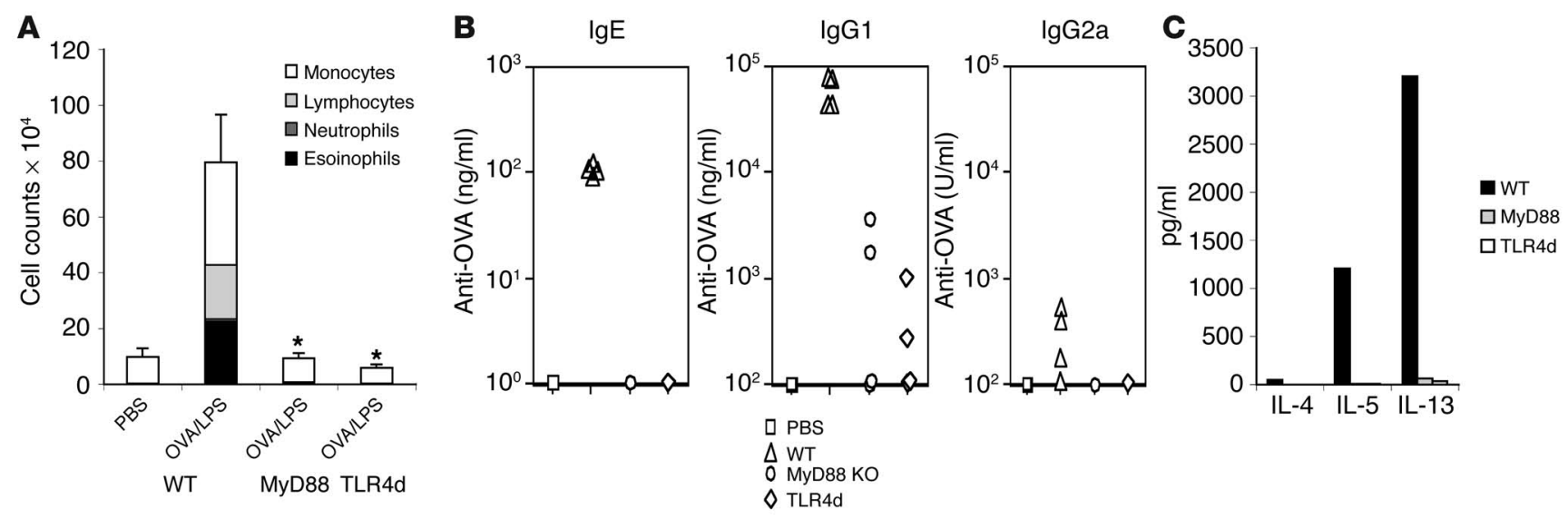

\section{Figure 1}

Th2 responses to intranasal protein antigen are MyD88 dependent. (A) BAL inflammatory cells of WT, MyD88-deficient, or TLR4d mice sensitized intranasally with OVA/LPS and WT mice sensitized with PBS. Exposed mice were then challenged 2 weeks later with inhaled OVA. Total bar height represents total BAL cell number, and error bars are based on total cell numbers. Stacked bars represent cell differential counts. $n=4$. ${ }^{*} P<0.05$, total BAL cell number from WT vs. MyD88-deficient and WT vs. TLR4d. One representative experiment of 4 is shown. (B) OVAspecific serum antibody responses by ELISA after challenge with OVA in WT, MyD88-deficient, and TLR4d mice sensitized as described in A. $P<0.05$, WT vs. MyD88-deficient and WT vs. TLR4d, for IgE and IgG1 responses. (C) Cytokine production from lung draining lymph nodes after challenge with OVA in WT, MyD88-deficient, and TLR4d mice sensitized as described in A.

pulmonary DCs is due to the inability of LPS to engage the MyD88independent pathway in the pulmonary environment. This MyD88dependent maturation is in contrast to the MyD88-independent maturation observed in BMDCs. Moreover, activated BMDCs can reconstitute Th2 responses in the pulmonary system of MyD88deficient recipients. In conclusion, the utilization of the innate TLR4/MyD88 components in the induction of Th2 responses is determined in a site-specific manner.

\section{Results}

Th2 responses to intranasal protein antigen are MyD88 dependent. We have previously developed a murine model for asthma based on sensitization to inhaled OVA in the absence of the traditionally used adjuvant aluminum hydroxide (25). Th2 responses generated in this model are characterized by airway inflammation, eosinophilia, mucus hypersecretion, IgE production, and production of predominantly Th2 cytokines. We have found that the generation of these Th2 responses required low doses of LPS acting in a TLR4-dependent manner (17). However, recent studies by others have demonstrated that in the absence of MyD88, Th2 responses are intact or augmented, which suggests that Th2 cells are activated in a MyD88independent fashion (12-15). Since LPS signals through both MyD88-dependent and MyD88-independent pathways, we sought to determine the roles of these pathways in the Th2 sensitization of mice to intranasal antigen. WT, TLR4-defective (C.C3H-TLR4LPS-d [TLR4d]), or MyD88-deficient mice were sensitized with PBS or OVA with low-dose LPS (OVA/LPS) intranasally.

Two weeks later, all groups of mice were challenged with intranasal OVA. As shown in Figure 1A, WT mice exposed to intranasal OVA/LPS compared with PBS demonstrated significant increases in total bronchoalveolar lavage (BAL) cell number with a characteristic eosinophilic infiltrate, indicative of a Th2 response. Moreover, both OVA-specific IgG1 and IgE were significantly induced in these mice (Figure 1B). As we have previously reported, these responses were significantly diminished in TLR4d mice, which demonstrates the LPS-specific TLR4 depen- dence of this Th2-mediated response. Surprisingly, however, MyD88-deficient mice also demonstrated diminished responses to OVA/LPS, with abrogation of IgE production and significant reductions in airway inflammation and eosinophilia (Figure 1, A and B). Generation of the Th2 cytokines IL-4, IL-5, and IL-13 in the draining lymph node was also significantly reduced in both TLR4- and MyD88-deficient mice (Figure 1C). These data indicate that the response to intranasal sensitization with OVA/LPS
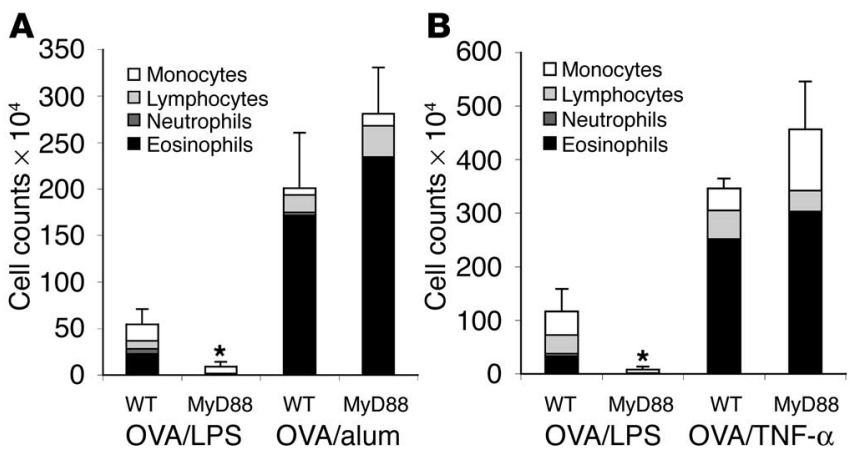

Figure 2

Th2-mediated lung inflammation can occur in the absence of MyD88. (A) BAL inflammatory cells of WT and MyD88-deficient mice sensitized with OVA/LPS intranasally or OVA/alum i.p. Exposed mice were then challenged 2 weeks later with inhaled OVA. Total bar height represents total BAL cell number at day 21 after challenge. Error bars are based on total cell numbers. Stacked bars represent cell differential counts. $n=4$. ${ }^{*} P<0.05$, WT vs. MyD88-deficient mice primed with OVA/LPS. One representative experiment of 3 is shown. (B) BAL inflammatory cells of WT and MyD88-deficient mice sensitized intranasally with OVA/LPS or OVA/TNF- $\alpha$. Exposed mice were then challenged 2 weeks later with inhaled OVA. Total bar height represents total BAL cell number at day 21 after challenge. Error bars are based on total cell numbers. Stacked bars represent cell differential counts. $n=4 .{ }^{*} P<0.05$, WT vs. MyD88-deficient mice primed with OVA/LPS. One representative experiment of 3 is shown. 
A

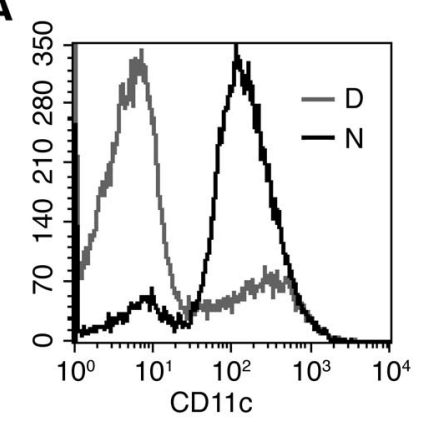

\begin{tabular}{|c|c|c|}
\hline \multirow[b]{2}{*}{$\begin{array}{c}\text { Experiment } \\
\text { no. }\end{array}$} & \multicolumn{2}{|c|}{$\begin{array}{c}\% \text { CD11c cells } \\
\left(\text { no. } \times 10^{5}\right)\end{array}$} \\
\hline & $\mathrm{N}$ & D \\
\hline 1 & $\begin{array}{c}40 \\
(1.36)\end{array}$ & $\begin{array}{c}5 \\
(0.75)\end{array}$ \\
\hline 2 & $\begin{array}{c}51 \\
(6.12)\end{array}$ & $\begin{array}{c}15 \\
(9.6)\end{array}$ \\
\hline 3 & $\begin{array}{c}78 \\
(4.52)\end{array}$ & $\begin{array}{c}19 \\
(1.54)\end{array}$ \\
\hline
\end{tabular}

Figure 3

Phenotype of lung cells in the absence of collagenase digestion. (A) Unstimulated lung cells harvested from WT mice with and without collagenase digestion and analyzed by FACS. Percentage yield and total number of CD11 $\mathrm{c}^{+}$cells obtained from resting lung by collagenase digestion (D) are shown compared with those from nondigested tissue (N). (B) Analysis of non-CD11 $\mathrm{C}^{+}$cell population from nondigested lung tissue by FACS. Expression of cell markers Mac1, B220, Gr1, $\gamma \delta$, NK, CD4, and CD8 on non-CD11 ${ }^{+}$cells is shown on forward scatter (Fsc) compared with an isotype control. Numbers indicate the percentage of live cells positive for the indicated cell marker.

is not only TLR4 dependent but, unlike previously studied Th2inducing antigens, is also MyD88 dependent.

Th2-mediated lung inflammation can occur in the absence of $M y D 88$. The loss of Th2 responses to intranasal OVA protein at low doses of LPS in MyD88-deficient mice, described above, could be due to a defect in sensitization, a defect in recruitment of inflammatory Th2 cells to the lung, or both. To test whether MyD88 has an effect on recruitment of inflammatory cells to the lung, we used $2 \mathrm{TLR} / \mathrm{MyD} 88$-independent sensitization protocols and analyzed the role of MyD88 in the challenge phase of the response. The first protocol entailed the commonly used i.p. sensitization with OVA in the presence of alum (26). Alum is a Th2 adjuvant devoid of microbial components. Thus, its action is considered to be independent of TLR signaling. We have previously demonstrated the induction of robust Th2 responses to OVA in alum in TLR4d mice, indicating that LPS was not required for these responses (17). WT and MyD88-deficient mice were sensitized with either OVA/alum i.p. or OVA/ LPS intranasally. Two weeks later all mice were challenged with intranasal OVA. As shown in Figure 2A, MyD88-deficient mice had eosinophilic inflammation in the lung similar to that in WT mice following i.p. sensitization with OVA/alum and intranasal OVA challenge. This is in agreement with prior studies demonstrating MyD88 independence of Th2 responses induced using OVA/alum sensitization (13).
Similar results were observed when mice were exposed to intranasal TNF- $\alpha$ with OVA antigen during sensitization. TNF- $\alpha$ is a major proinflammatory cytokine secreted in response to TLR engagement that is capable of inducing DC maturation and migration (27-30). While TNF- $\alpha$ secretion has been reported to be MyD88 dependent, TNF- $\alpha$ signaling can occur independently of TLRs and MyD88 (19, 20, 24). Consistent with this, we have previously found that TNF- $\alpha$ can reconstitute Th2 responses in TLR4d mice (17). Therefore we reasoned that TNF- $\alpha$ should also be able to restore Th2 responses to intranasal OVA in MyD88-deficient mice. WT and MyD88-deficient mice were sensitized with OVA/TNF- $\alpha$ intranasally and then challenged 2 weeks later with intranasal OVA in the absence of TNF- $\alpha$. Potent Th2 responses were generated in MyD88-deficient mice when TNF- $\alpha$ was included during the priming phase, inducing the generation of a robust eosinophilic infiltrate in the lung (Figure 2B). Taken together, these data indicate that the loss of Th2 responses to low-dose LPS in MyD88-deficient mice results from a failure to prime rather than an intrinsic defect in the ability to recruit Th2 cells to the lung.

Activation of pulmonary DCs requires MyD88. DC surface expression of MHC class II and B7 costimulatory molecules is essential for naive $\mathrm{T}$ cell activation and thus for induction of Th2 responses (30). Prior studies have demonstrated that in response to LPS, BMDCs from MyD88deficient mice retain the capacity to upregulate both these molecules at levels similar to those of WT BMDCs (21). However, given the loss of Th2 responses to intranasal OVA in MyD88-deficient mice, we sought to determine the capacity of pulmonary DCs to upregulate expression of these molecules in response to LPS. We have previously demonstrated the preferential uptake and presentation of intranasally administered antigen to antigen-specific $\mathrm{T}$ cells by pulmonary cells expressing the DC marker CD11c (31). In order to maximize the yield of CD11 $\mathrm{c}^{+}$cells and to minimize potential in vitro activation, we isolated lung cells in the absence of collagenase digestion. Animals used for our experiments were also kept in clean facilities to minimize potential induction of pulmonary activation by environmental microbes. The isolation of lung cells from these animals in the absence of collagenase leads to a greater percentage of $\mathrm{CD} 11 \mathrm{c}^{+}$cells as compared with isolation with collagenase digestion, although the total numbers of $\mathrm{CD} 11 \mathrm{c}^{+}$ cells obtained are comparable (Figure 3A). Our data agree with others' in that very few inflammatory cells were observed in the resting lung $(25,32-35)$. The non-CD11 $\mathrm{c}^{+}$cells that we obtained by this method included a Mac1 $1^{+}$cell population, as previously described (31) (Figure 3B); few B cells, granulocytes, T cells, NK cells, and $\gamma \delta$ cells were observed (Figure $3 \mathrm{~B}$ ).

In order to examine the response of pulmonary DCs to LPS in vivo, WT and MyD88-deficient mice were subjected to intranasal administration of OVA/LPS. Lung cells were harvested, and the phenotype of $\mathrm{CD} 11 \mathrm{c}^{+}$lung cells was observed. Expression of both MHC class II and CD86 on CD11 $\mathrm{c}^{+}$lung cells was increased in 

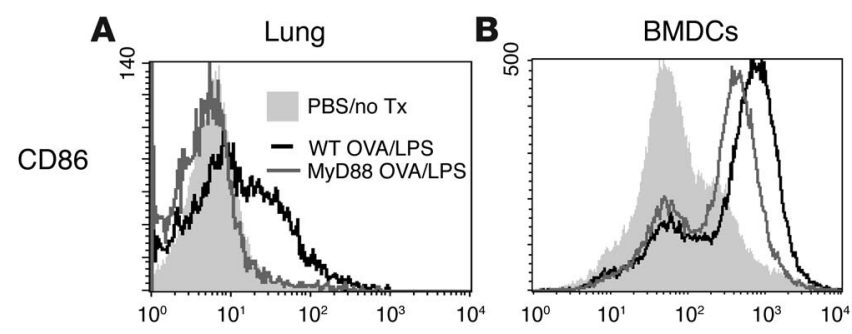

MHC II
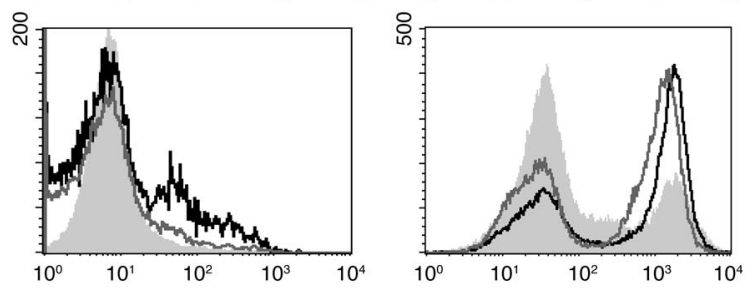

WT mice, compared with PBS controls, in response to OVA/LPS (Figure 4A). However, MyD88-deficient mice failed to upregulate MHC class II and CD86 on CD11 $\mathrm{c}^{+}$pulmonary DCs following exposure to intranasal OVA/LPS (Figure 4A). Consistent with previous studies, CD11 $\mathrm{c}^{+}$BMDCs from both WT and MyD88deficient mice did upregulate MHC class II and CD86 in response to OVA/LPS in vitro (Figure 4B). These results suggested that the inability to induce Th2 responses in the absence of MyD88 is due in part to defective pulmonary DC maturation.

One possible explanation for the observed divergence in response between pulmonary DCs and BMDCs lies in differences between in vivo and in vitro modes of stimulation. Thus, to more directly compare the effects of LPS on pulmonary DCs versus BMDCs, both CD $11 \mathrm{c}^{+}$lung cells and BMDCs from WT and MyD88-deficient mice were stimulated in vitro with different doses of LPS, and CD86 expression was determined. Our findings indicate that WT pulmonary DCs were less responsive to LPS than BMDCs in vitro (Figure 5A). Moreover, in accord with our in vivo studies and in contrast to the results with BMDCs, increased CD86 expression on pulmonary DCs was MyD88 dependent (Figure 5, B and C). In summary, these data indicate that pulmonary DC activation is MyD88 dependent.

Pulmonary type I IFN expression is defective in response to LPS. LPS engagement of the MyD88-dependent pathway is characterized by induction of inflammatory cytokines such as TNF- $\alpha$. LPS engagement of the MyD88-independent pathway is characterized centrally by induction of IFN- $\beta$. More generally, TLRs can be divided into subgroups based on their ability to induce IFN- $\beta$ (36). In particular, TLR4 and TLR9 have been demonstrated to possess the capacity to induce IFN- $\beta$ production. These TLRs can

\section{Figure 5}

MyD88-deficient pulmonary DCs demonstrate defective activation in response to LPS in vitro. (A) Change in CD86 expression at 24 hours on CD11 $\mathrm{C}^{+}$lung cells from WT mice and CD11 $\mathrm{C}^{+}$BMDCs generated from WT mice responding to stimulation with varying doses of LPS in vitro. Bar heights represent the mean of 3 independent experiments. (B) Change in CD86 expression at 24 hours on CD11 $\mathrm{c}^{+}$BMDCs generated from WT and MyD88-deficient mice. (C) Change in CD86 expression at 24 hours on CD11 $\mathrm{c}^{+}$lung cells from WT and MyD88deficient mice. ${ }^{*} P<0.05$, WT vs. MyD88-deficient lung cells and WT lung cells vs. WT BMDCs.

\section{Figure 4}

Pulmonary DCs are not activated by LPS in the absence of MyD88 in vivo. (A) MHC class II and CD86 FACS analysis, at 48 hours, of CD11 $\mathrm{C}^{+}$lung cells from WT and MyD88-deficient mice sensitized to OVA/LPS or PBS intranasally. (B) MHC class II and CD86 FACS analysis of CD11 $\mathrm{C}^{+}$BMDCs generated from WT and MyD88-deficient mice pulsed with OVA/LPS overnight or left untreated (no Tx).

be further divided based on the adaptor molecule responsible for such IFN- $\beta$ production. IFN- $\beta$ induction by $C \mathrm{pG}$ engagement of TLR9 has been demonstrated to be MyD88 dependent. In contrast, IFN- $\beta$ induction by LPS engagement of TLR 4 has been demonstrated to be MyD88 independent, mediated instead by the recently identified adaptor molecule TIR domain-containing adaptor inducing IFN- $\beta$ (TRIF) $(23,24)$. In this regard, IFN- $\beta$ expression has been used as a measure of activation of the MyD88-independent pathway subsequent to the engagement of TLR4 by LPS. LPS engagement of the TLR4/MyD88-independent pathway has been previously considered to be sufficient for upregulation of costimulatory molecules on the surface of APCs. Recent studies have suggested that such upregulation is dependent on LPS engagement of TRIF and subsequent downstream activation of IFN- $\beta$ (37). Given that our studies demonstrate the defective upregulation of costimulatory molecules by pulmonary APCs in the absence of MyD88 in response to LPS, we sought to determine, through an assessment of IFN- $\beta$ induction, whether the MyD88 dependence of pulmonary Th2 responses was a consequence of defective engagement of the MyD88-independent pathway by LPS in the lung. Thus, WT mice were exposed to PBS, LPS,

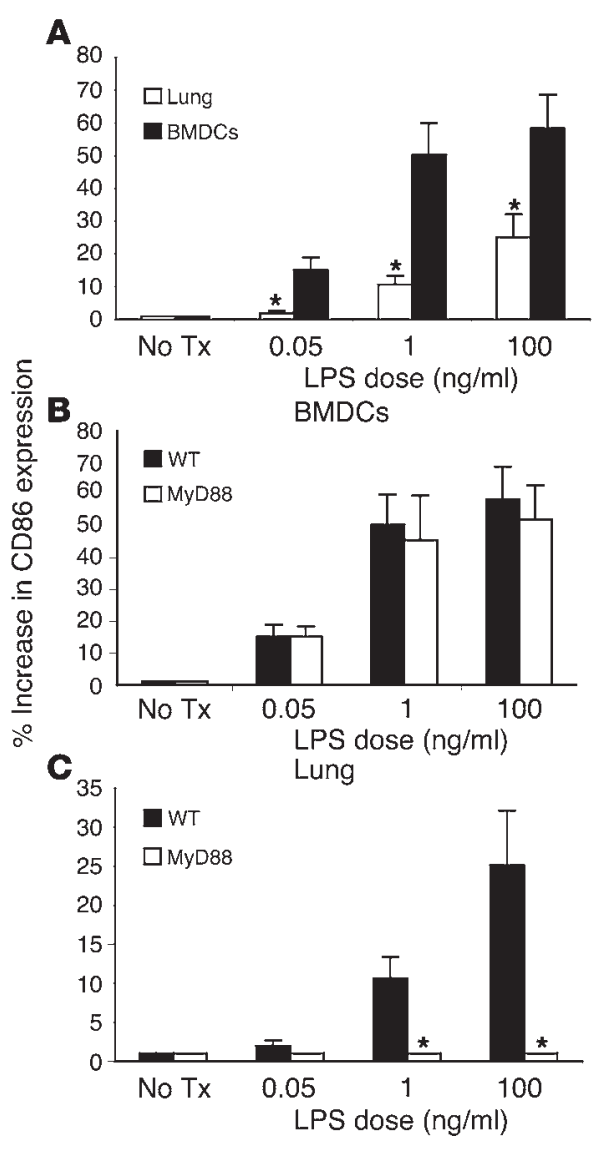



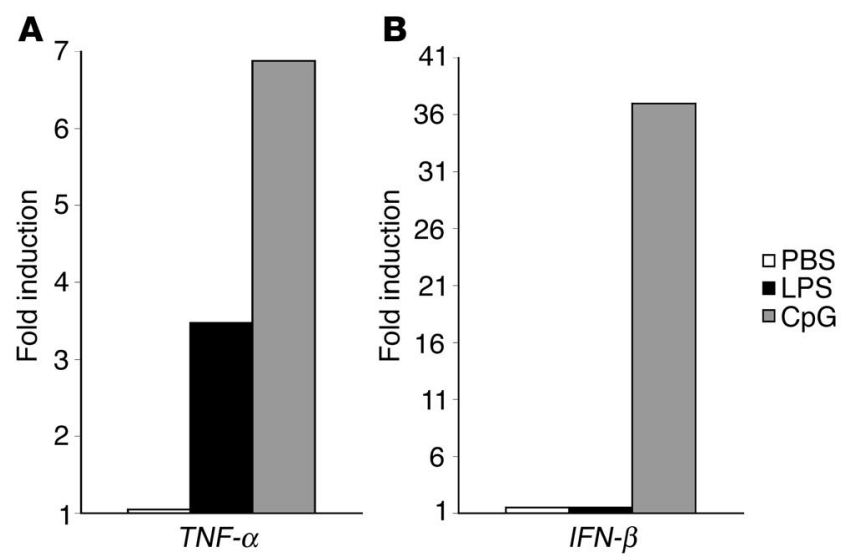

Figure 6

IFN- $\beta$ is not induced in response to LPS exposure in the lung. WT mice were exposed to PBS, LPS, and CpG. Lung cells were harvested 18 hours after exposure and lysed, and RNA was extracted. Five micrograms of each RNA sample was analyzed by RNase protection assay using a RiboQuant mCK-3b probe. Fold induction of (A) TNF- $\alpha$ and (B) IFN- $\beta$ is shown relative to PBS.

or $\mathrm{CpG}$, and the expression of TNF- $\alpha$ and $I F N-\beta$ in the lung was determined. Whereas exposure to both LPS and CPG led to the induction of TNF- $\alpha$ (Figure 6A), LPS was unable to induce IFN- $\beta$ expression in the lung (Figure 6B). In contrast, induction of IFN- $\beta$ was intact in response to the TLR9 ligand CPG. These studies suggest defective engagement of the MyD88-independent pathway by LPS in the pulmonary environment.

Mature BMDCs can reconstitute Th2 responses in MyD88-deficient mice. Our data indicate that the failure to induce Th2 responses in MyD88-deficient mice may be in part due to defective DC maturation. In order to further examine the role of DC activation in the induction of pulmonary Th2 responses, we examined whether transfer of activated DCs would reconstitute Th2 responses in MyD88-deficient mice. BMDCs generated from WT mice were cultured with or without OVA/LPS in vitro and transferred intranasally to WT or MyD88-deficient recipients. As seen in Figure 2B, these BMDCs express increased levels of MHC II and CD86 in response to OVA/LPS. Transfer of OVA/LPS-activated BMDCs into WT mice induced a robust Th2 eosinophilic inflammatory response (Figure 7). Furthermore, a comparable response was induced when activated BMDCs were transferred into MyD88deficient mice. Transfer of WT BMDCs not exposed to OVA to WT recipients did not induce Th2 inflammatory responses. These data suggest that MyD88-deficient mice exposed to intranasal antigen do possess the capacity to generate Th2 responses in the presence of activated DCs.

Th1 responses induced by intranasal OVA with increased LPS dose are also MyD88 dependent. Several studies have shown that Th1 responses induced by immunization with potent Th1-inducing agents such as Toxoplasma gondii were completely MyD88 dependent (15). In the absence of MyD88, these Th1-inducing agents elicit Th2 responses instead. These data suggest that potent immunogens can elicit specifically a Th2 response in the absence of MyD88. We have previously observed that the differentiation of Th2 versus Th1 responses is dependent on LPS dose, with Th2 responses being induced with OVA at low doses of LPS and Th1 responses being observed with OVA at high doses of LPS.
Therefore, in order to determine whether default pulmonary Th2 responses could be induced with OVA at high doses of LPS in the absence of MyD88, we examined the effect of increased LPS dosage on the induction of OVA-specific responses in MyD88-deficient mice. Consistent with our previous studies, WT mice demonstrated a characteristic Th1 response to OVA when high-dose LPS was present during priming, with a lack of eosinophilic but a robust neutrophilic inflammatory response (Figure 8). However, the pulmonary inflammatory response induced by OVA at high doses of LPS was significantly diminished in MyD88-deficient mice. Thus, both Th1 and Th 2 responses elicited by intranasal antigen are dependent on MyD88.

Th2 responses to i.p. protein antigen are TLR 4 and MyD88 independent. Studies demonstrating default to Th2 responses in the absence of MyD88 used different routes of sensitization, including s.c. and i.p. antigen delivery (12-15). Thus, the requirement for MyD88 may depend on the initial site of antigen exposure. We have demonstrated in this study that Th2 responses are intact when MyD88-deficient mice are sensitized i.p. with OVA in alum. However, alum is considered to exert its effects independently of TLR signaling. Moreover, we observed the generation of Th2 responses to intranasal sensitization with OVA/LPS in the absence of alum in WT mice. Thus, in order to further determine whether a differential site requirement for MyD88 exists, we compared the pulmonary responses of WT and MyD88-deficient mice sensitized to OVA/LPS either i.p. (in the absence of alum) or intranasally. In contrast to MyD88-deficient mice sensitized intranasally, MyD88-deficient mice sensitized i.p. with OVA/LPS demonstrated no defect in their ability to induce Th2 responses in the airways (Figure 9A). In addition, TLR4d mice responding to i.p. sensitization demonstrated a Th2 response at levels similar to those seen in WT mice (Figure 9B). Thus, the TLR4/MyD88 dependence of pulmonary Th2 responses to OVA appears to be specific to the sensitization site.

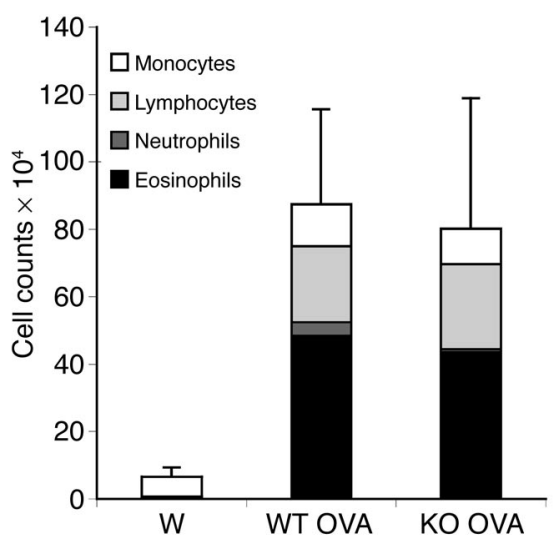

\section{Figure 7}

Mature BMDCs can reconstitute Th2 responses in MyD88-deficient mice. BAL inflammatory cells of WT and MyD88-deficient mice given OVA/LPS-pulsed BMDCs intranasally and challenged intranasally with OVA after 2 weeks. Total bar height represents total BAL cell number at day 21. Error bars are based on total cell numbers. Stacked bars represent cell differential counts. $n=4$. W, unpulsed WT BMDCs to WT recipient; WT OVA, OVA/LPS-pulsed WT BMDCs to WT recipient; KO OVA, OVA/LPS-pulsed WT BMDCs to MyD88deficient recipient. $P=0.82$, WT OVA vs. KO OVA. One representative experiment of 3 is shown. 


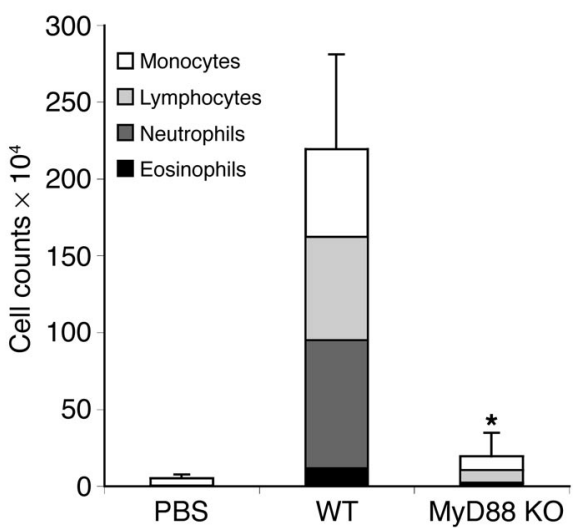

Figure 8

High-dose LPS response is MyD88 dependent. BAL inflammatory cells of WT and MyD88-deficient mice sensitized with OVA/high-dose LPS intranasally. Exposed mice were challenged 2 weeks later with inhaled OVA. Total bar height represents total BAL cell number at day 21 after challenge. Error bars are based on total cell numbers. Stacked bars represent cell differential counts. ${ }^{*} P<0.05$, OVA/highdose LPS-primed WT vs. MyD88-deficient mice. One representative experiment of 3 is shown.

The activation status of an APC is known to be critical for the induction of $\mathrm{CD}^{+} \mathrm{T}$ cell responses. Thus, we considered that the differential requirement of the pulmonary versus the peritoneal site for TLR4 and MyD88 in the induction of Th2 responses to OVA may result from a possibly differential baseline activation status of APCs resident at these sites. Therefore, we compared the maturational status of APCs at these 2 sites. Pulmonary and peritoneal APCs were obtained without any prior in vivo manipulation and examined for expression of MHC class II, CD86, and CD40. Whereas WT pulmonary APCs demonstrated little expression of any of these molecules (Figure 10A), both WT and MyD88-deficient peritoneal APCs expressed relatively high levels of all 3 molecules (Figure 10, B and C). Thus, peritoneal APCs appear to possess the requisite surface molecules for $\mathrm{CD}^{+} \mathrm{T}$ cell activation prior to antigen exposure.

\section{Discussion}

Identification of the crucial role of the innate immune recognition apparatus in the initiation of adaptive Th 1 immune responses has in large part centered around studies involving MyD88deficient mice. Models incorporating potent Th1-inducing antigens such as T. gondii and Leishmania major (in C57BL/6 mice) have demonstrated a Th1-to-Th2 switch in the absence of MyD88 $(14,15)$. Protocols inducing a mixed Th1/Th2 response, such as those involving CFA, demonstrate loss of Th1 responses and maintenance of Th2 responses $(12,13)$. Moreover, Th2 responses to the helminth-derived stimulus schistosome egg antigen are also maintained in MyD88-deficient mice (15). The coupling of these results to our initial findings of a TLR4-dependent Th2 response to inhaled antigen was strongly suggestive of a MyD88independent mechanism for induction of allergic Th2 responses to LPS. However, our studies demonstrate a critical role for the MyD88-dependent pathway in the LPS-induced sensitization of Th2 responses to intranasal protein antigen and suggest both APC-specific and site-specific utilization of the innate system in the induction of adaptive Th2 immunity. Moreover, our studies identify a critical role for the MyD88-dependent pathway in the endotoxin sensitization of allergic responses.

$\mathrm{APC}$ maturation is essential for $\mathrm{CD}^{+} \mathrm{T}$ cell activation. Control of this maturation process is an integral component of maintaining immune homeostasis. TLRs have been shown to play a major role in this maturation program, a role that includes both the upregulation of costimulatory molecules and the induction of inflammatory cytokines and type I IFN. Previous studies on BMDCs and peritoneal APCs have indicated that whereas MyD88 is required for induction of inflammatory cytokines such as TNF- $\alpha$, it does not appear to be required for upregulation of costimulatory molecules on the surface of these APCs in response to LPS signaling through TLR4. Upregulation of costimulatory molecules in response to LPS has been suggested to be dependent instead on the adaptor molecule TRIF, which mediates the MyD88-independent pathway (37). Upregulation by TRIF in this regard has been further postulated to be mediated by type I IFN production. At the same time, signaling through several other TLRs has been demonstrated to upregulate costimulatory molecules in a MyD88-dependent manner $(7,21,37)$. Our in vivo and in vitro studies suggest that upregulation of costimulatory molecules on the surface of pulmonary APCs via TLR4 is defective in the absence of MyD88, in contrast to observations of MyD88-deficient BMDCs. Thus, the defect in Th2 induction observed in our studies likely results from defective activation of MyD88-deficient pulmonary APCs. This is supported by the ability of mature BMDCs to reconstitute Th2 responses in MyD88-deficient mice. Moreover, the dependence of pulmonary APC activation on MyD88 appears to result from a defect in LPS engagement of the type I IFN-producing MyD88independent/TRIF-dependent pathway in the pulmonary environ-

\section{Figure 9}

Th2 responses to i.p. protein antigen are TLR4 and MyD88 independent. BAL inflammatory cells of WT mice and MyD88-deficient mice (A) or TLR4d mice (B) sensitized i.p. with OVA/LPS. Exposed mice were challenged 2 weeks later with inhaled OVA. Total bar height represents total BAL cell number at day 21 after challenge. Error bars are based on total cell numbers. Stacked bars represent cell differential counts. $n=4 . P=0.68$, WT vs. TLR4d; $P=0.33$, WT vs. MyD88-deficient.
A

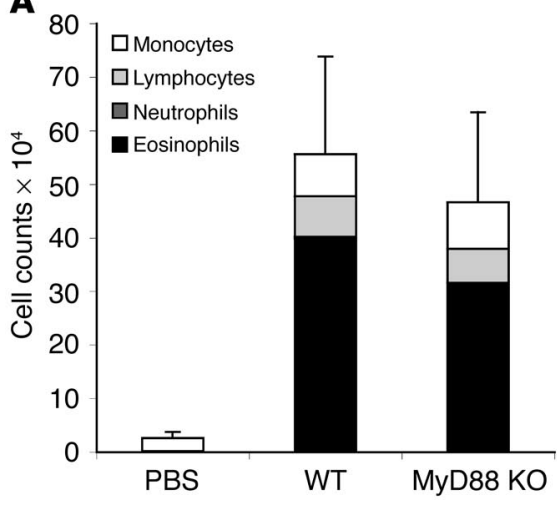

B

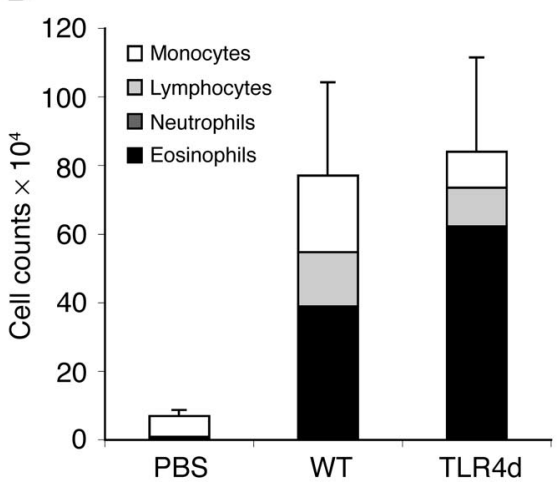



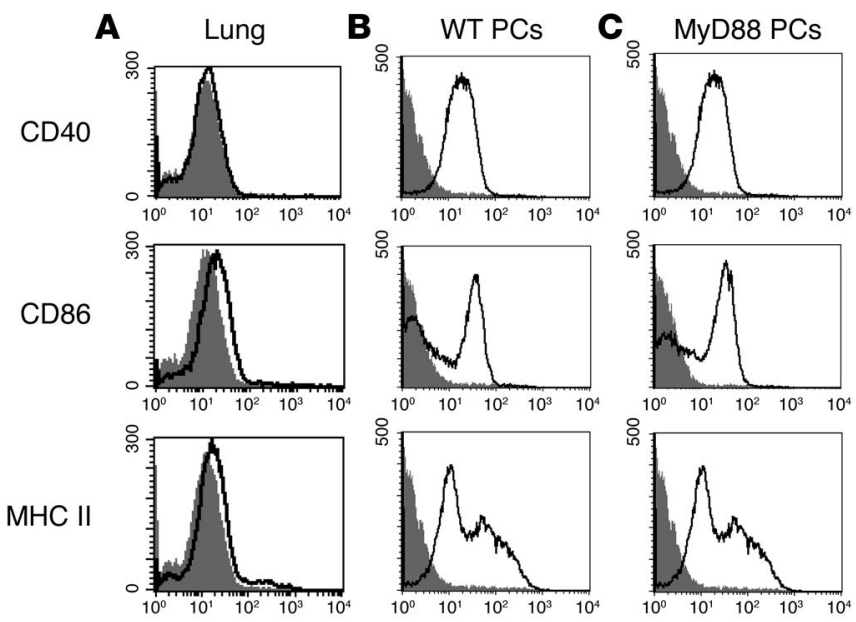

MHC II

\section{Figure 10}

Pulmonary APCs demonstrate low maturational status compared with peritoneal APCs. Unstimulated lung cells harvested from WT mice $(\mathbf{A})$ and unstimulated peritoneal cells (PCs) from WT mice (B) and MyD88-deficient mice (C), analyzed by FACS. MHC class II, CD86, and CD40 expression on live cells is shown compared with isotype control (shaded).

ment (37). Our studies suggest that APCs, depending on their mode of generation in vitro or resident location in vivo, have different capacities to engage MyD88-dependent and MyD88-independent pathways in response to specific TLR engagement, with significant consequences for functional immune responses.

An important note thus should be made about the generalizability of APC function in the study of adaptive immunity. BMDCs have been used as a model for in vivo APC function with significant utility. In addition, BMDC transfers have been used as a model for pulmonary APCs in the study of allergic responses. APCs do share certain common traits with BMDCs. However, our findings in conjunction with others' suggest that pulmonary APCs differ in certain significant respects from BMDCs and APCs at other sites. Specialized characteristics of pulmonary APCs, possibly determined by a combination of intrinsic developmental control and microenvironmental effects, should be considered in the evaluation of pulmonary APC function in both innate and adaptive immune responses.

Reconciliation of our findings with previous accounts of MyD88independent Th2 responses could derive from differences in the nature or strength of the antigenic stimulus or from differences in the site of antigen delivery. One recent study has demonstrated the induction of Th2 responses to flagellin, a TLR5 ligand that signals exclusively through MyD88 (38). Flagellin and LPS thus may be among a subset of pathogen-associated molecular patterns uniquely capable of inducing Th2 responses. However, since LPS has been demonstrated in other studies to induce Th2 responses in the absence of MyD88, the type of antigen used in our studies is unlikely to account for our findings (12). Moreover, we have found that increased LPS dose does not induce a Th2 default response in our system; this indicates that the strength of signal is unlikely to be playing a role. Site specificity is a more likely proposition. Previous studies of Th2 default in the absence of MyD88 involved extrapulmonary administration of antigen. However, our sensitization protocol involved direct intranasal administration of antigen. In support of the essential role of site specificity, we have found that epicutaneous Th2 sensitization to OVA also appears to be significantly impaired in the absence of MyD88 (C.A. Herrick et al., unpublished data). In contrast, induction of Th2 responses to i.p. administration of protein antigen is both TLR4 and MyD88 independent, consistent with prior studies of antigen sensitization at this site. Interestingly, the MyD88 independence of peritoneal Th2 sensitization occurs even in the absence of alum administration. One canonical mouse model of asthma is based on i.p. sensitization with OVA/alum (26). The difference in site requirements for induction of Th2 responses that was observed in our studies does suggest some limitation in the use of this i.p. model for allergic disease and demonstrates the importance of using more natural sites of sensitization in elucidating mechanisms for the induction of pulmonary Th2-mediated allergic responses.

Uncontrolled or dysregulated immune responses can be as harmful to a host as deficient responses. Generation of an appropriate immune response to the right antigen is thus a crucial component of effective immunity. Mucosal sites such as the lung are at particular risk for inappropriate $\mathrm{T}$ cell activation, given their continual exposure to an onslaught of environmental antigens. Given the increased potential for overstimulation at these sites, tighter control of responses to antigenic stimuli is likely required, in comparison with that at sites that are less accessible to environmental antigens. Several layers of control likely exist to maintain homeostasis at these sites, including the maintenance of APCs in an immature state, as well as active suppressive mechanisms characteristic of specialized mucosal microenvironments, such as regulatory $\mathrm{T}$ cells and antiinflammatory cytokines. Both of these mechanisms for maintenance of peripheral tolerance can be modulated by the innate immune system in general and MyD88 in particular $(39,40)$. The innate immune system likely developed primarily to protect the host upon exposure to infectious non-self. The central role of the TLR adaptor molecule MyD88 in pulmonary Th2 immunity suggests that aberrant Th2-mediated allergic responses likely follow from the commandeering of the innate immune system to disrupt natural mechanisms of homeostasis at these sites. Thus, targeting innate mechanisms for control of adaptive Th2 immunity may have a significant impact on the alleviation of Th2-mediated pathological states such as asthma and allergy.

\section{Methods}

Animals. MyD88-/- mice were generated as previously described (41) and kindly provided on BALB/c and B6/129 backgrounds by R. Medzhitov (Yale University School of Medicine). MyD88/- mice on the BALB/c background were backcrossed at least 6 times before use. BALB/c and B6/129PF2 mice purchased from The Jackson Laboratory were used as controls for KO mice on BALB/c and B6/129 backgrounds, respectively. TLR4d mice were also purchased from The Jackson Laboratory. Mice were used at 6-10 weeks of age. All animal studies were performed in accordance with the guidelines of the Yale University Institutional Animal Care and Use Committee.

Sensitization protocols. For intranasal sensitization, mice were lightly anesthetized with methoxyflurane and subjected to intranasal delivery of $100 \mu \mathrm{g}$ OVA protein (Grade V; Sigma-Aldrich) in $50 \mu \mathrm{l}$ PBS on days 0 , 1 , and 2 as previously described (25). Two micrograms of recombinant TNF- $\alpha$ (R\&D Systems Inc.) was coadministered on day 1 (Figure 2B). For i.p. sensitization, mice were injected with $100 \mu \mathrm{g}$ OVA in $2 \mathrm{mg}$ aluminum hydroxide (Pierce Chemical Co.) as previously described (17) or with $100 \mu \mathrm{g}$ OVA in $200 \mu \mathrm{l}$ PBS on days 0,1 , and 2 in the absence of alum. For low- 
dose LPS treatment, 0.008-0.12 $\mu \mathrm{g}$ E. coli LPS O55:B5 (Sigma-Aldrich) was used on each day of sensitization. For high-dose LPS treatment, 10-40 $\mu \mathrm{g}$ LPS was used.

Challenge protocol. For all sensitization protocols, mice were lightly anesthetized with methoxyflurane and challenged intranasally with $25 \mu \mathrm{g}$ OVA in $50 \mu \mathrm{l}$ PBS on days 14,15,18, and 19, and sacrificed on day 21 .

$B A L$ analysis. BAL fluid was obtained by tracheal infusion of $3 \mathrm{ml} P B S$ as previously described (42). BAL cells were treated with rbc lysis medium (1.6 g $\mathrm{NH}_{4} \mathrm{Cl}, 0.2 \mathrm{~g} \mathrm{KHCO}_{3}$, and $0.03 \mathrm{~g}$ EDTA) for 5 minutes. Cells were then washed, counted, and spun onto slides. Slides were stained with H\&E (DiffQuik; Dade Behring Inc.), and differential cell counts were obtained based on morphology and staining characteristics.

Serum antibody detection. Serum was obtained by cardiac puncture on day 21 for measurement of OVA-specific IgE, IgG1, and IgG2a antibodies by ELISA as previously described $(25,43)$. IgE standard was a kind gift of E. Gelfand (National Jewish Center for Immunology and Respiratory Medicine, Denver, Colorado, USA). Levels of detection were $5 \mathrm{ng} / \mathrm{ml}$ (IgE), $125 \mathrm{ng} / \mathrm{ml}$ (IgG1), and $81 \mathrm{U} / \mathrm{ml}$ (IgG2a).

Lymph node cytokine production. Mediastinal lymph node cells were isolated on day 21 after challenge and stimulated in vitro with $200 \mu \mathrm{g} / \mathrm{ml}$ OVA and syngeneic T cell-depleted splenocytes. Cytokines in culture supernatants were measured using commercially available ELISA kits (R\&D Systems Inc.). Levels of detection were $25 \mathrm{pg} / \mathrm{ml}$ (IL-4), $125 \mathrm{pg} / \mathrm{ml}$ (IL-5), and $31.2 \mathrm{pg} / \mathrm{ml}$ (IL-13).

Lung cell preparation. Mice were subjected to intranasal administration of $100 \mu \mathrm{g}$ OVA with LPS on day 0 and sacrificed on day 2 . Lungs were perfused with $20 \mathrm{ml}$ PBS through the right ventricle. Whole lungs were recovered. For physical disruption, we used either a metal sieve or glass slides. Cell strainers were used to remove debris. Cell strainers (BD Falcon; BD Biosciences - Discovery Labware) were $100 \mu \mathrm{m}$ in diameter. Cell suspensions were subjected to centrifugation over a Ficoll gradient. For digestion experiments, lung pieces were incubated with $150 \mathrm{U} / \mathrm{ml}$ collagenase (CLS-1; Worthington Biochemical Corp.) and $20 \mu \mathrm{g} / \mathrm{ml}$ type IV DNase I (Sigma-Aldrich) at $37^{\circ} \mathrm{C}$ for 20 minutes prior to disruption through a metal sieve. For in vitro experiments, lung cells were harvested from unstimulated mice and exposed to varying concentrations of LPS overnight (12-24 hours). Lung cells were then subjected to FACS analysis (BD Biosciences).

$B M D C$ preparation and sensitization. BMDCs were cultured as previously described (44). Briefly, bone marrow cells were isolated by perfusion of the femur and tibia. Cells were cultured in RPMI 1640 (Invitrogen Corp.) in the presence of $1 \%$ culture supernatant from a cell line transfected with the murine GM-CSF gene. The culture medium was supplemented with FCS, penicillin, streptomycin, and L-glutamine. After 6 days, cells were harvested and pulsed with $100 \mu \mathrm{g} / \mathrm{ml}$ OVA containing $1 \mathrm{ng} / \mathrm{ml}$ LPS or with varying concentrations of LPS overnight. Mice were lightly anesthetized with methoxyflurane, and then $1 \times 10^{6}$ OVA-pulsed BMDCs in $50 \mu \mathrm{l}$ PBS were administered intranasally. BMDCs were subjected to FACS analysis.

Peritoneal cell preparation. Peritoneal cells were obtained without thioglycollate induction. The peritoneal cavity was infused with 5-10 $\mathrm{ml}$ PBS. The fluid-filled cavity was gently shaken, and fluid was withdrawn. Cells were washed and subjected to FACS analysis.

FACS analysis. BMDCs, lung cells, and peritoneal cells were resuspended in PBS $/ 5 \%$ FCS containing $0.01 \%$ sodium azide. Cells were incubated with anti-FcR in combination with mouse Ig for 20 minutes on ice. Cells were then stained with antibodies against CD11c, CD86, CD40, MHC class II, B220, Gr1, $\gamma \delta, \mathrm{NK}, \mathrm{CD} 4, \mathrm{CD} 8$, and isotype control (BD Biosciences - Pharmingen) for 30 minutes on ice. Cells were analyzed by FACScan in association with CellQuest software (BD Biosciences).

RNase protection assay. Lung cells were harvested from mice 18 hours after exposure to PBS, $0.12 \mu \mathrm{g}$ LPS, and $10 \mu \mathrm{g}$ CpG DNA oligonucleotides. Lung cells were then lysed in TRIzol reagent (GIBCO BRL; Invitrogen Corp.). Cytokine RNA levels were analyzed by RNase protection assay using the RiboQuant mCK-3b template (BD Biosciences - Pharmingen) according to the manufacturer's protocol. Five micrograms of total RNA was used in each reaction. Transcript levels for the L32 housekeeping gene were used as a control for equal loading.

Statistical significance. Statistical significance was determined using Student's $t$ test.

\section{Acknowledgments}

The authors would like to thank Patricia Ranney for technical assistance. This work was supported by NIH grants HL54450 and HL56389, and Medical Scientist Training Program grant 5 T32 GM07205. S.L. Constant is funded by a grant from the American Heart Association.

Received for publication June 17, 2004, and accepted in revised form November 9, 2004.

Address correspondence to: Damani Piggott, Yale University School of Medicine, Section of Immunobiology, 300 Cedar Street, The Anlyan Center S555, PO Box 208011, New Haven, Connecticut 06520, USA. Phone: (203) 785-5391; Fax: (203) 737-1765; E-mail: piggotda@biomed.med.yale.edu.
1. Constant, S.L., and Bottomly, K. 1997. Induction of Th1 and Th2 CD4+ T cell responses: the alternative approaches. Annu. Rev. Immunol. 15:297-322.

2. Eisenbarth, S.C., Piggott, D.A., and Bottomly, K. 2003. The master regulators of allergic inflammation: dendritic cells in Th2 sensitization. Curr Opin. Immunol. 15:620-626.

3. Lenschow, D.J., Walunas, T.L., and Bluestone, J.A. 1996. CD28/B7 system of T cell costimulation. Annu. Rev. Immunol. 14:233-258.

4. Sharpe, A.H., and Freeman, G.J. 2002. The B7CD28 superfamily. Nat. Rev. Immunol. 2:116-126.

5. Janeway, C.A., Jr., and Medzhitov, R. 2002. Innate immune recognition. Annu. Rev. Immunol. 20:197-216.

6. Janeway, C.A., Jr. 1989. Approaching the asymptote? Evolution and revolution in immunology. Cold Spring Harb. Symp. Quant. Biol. 54:1-13.

7. Takeda, K., Kaisho, T., and Akira, S. 2003. Toll-like receptors. Annu. Rev. Immunol. 21:335-376.

8. Medzhitov, R., et al. 1998. MyD88 is an adaptor protein in the hToll/IL-1 receptor family signaling pathways. Mol. Cell. 2:253-258.

9. Muzio, M., Natoli, G., Saccani, S., Levrero, M., and Mantovani, A. 1998. The human toll signaling pathway: divergence of nuclear factor kappaB and $\mathrm{JNK} / \mathrm{SAPK}$ activation upstream of tumor necrosis factor receptor-associated factor 6 (TRAF6). J. Exp. Med. 187:2097-2101.

10. Yamamoto, M., Takeda, K., and Akira, S. 2004. TIR domain-containing adaptors define the specificity of TLR signaling. Mol. Immunol. 40:861-868.

11. Barton, G.M., and Medzhitov, R. 2003. Toll-like receptor signaling pathways. Science. 300:1524-1525.

12. Kaisho, T., et al. 2002. Endotoxin can induce MyD88-deficient dendritic cells to support $\mathrm{T}(\mathrm{h}) 2$ cell differentiation. Int. Immunol. 14:695-700.

13. Schnare, M., et al. 2001. Toll-like receptors control activation of adaptive immune responses. Nat. Immunol. 2:947-950.

14. Muraille, E., et al. 2003. Genetically resistant mice lacking MyD88-adapter protein display a high susceptibility to Leishmania major infection associated with a polarized Th2 response. J. Immunol.
170:4237-4241.

15. Jankovic, D., et al. 2002. In the absence of IL-12, CD4(+) T cell responses to intracellular pathogens fail to default to a Th2 pattern and are host protective in an IL-10(-/-) setting. Immunity. 16:429-439.

16. Barton, G.M., and Medzhitov, R. 2002. Control of adaptive immune responses by Toll-like receptors. Curr. Opin. Immunol. 14:380-383.

17. Eisenbarth, S.C., et al. 2002. Lipopolysaccharideenhanced, toll-like receptor 4-dependent T helper cell type 2 responses to inhaled antigen. J. Exp. Med. 196:1645-1651.

18. Dabbagh, K., Dahl, M.E., Stepick-Biek, P., and Lewis, D.B. 2002. Toll-like receptor 4 is required for optimal development of Th2 immune responses: role of dendritic cells. J. Immunol. 168:4524-4530.

19. Kawai, T., Adachi, O., Ogawa, T., Takeda, K., and Akira, S. 1999. Unresponsiveness of MyD88-deficient mice to endotoxin. Immunity. 11:115-122.

20. Kawai, T., et al. 2001. Lipopolysaccharide stimulates the MyD88-independent pathway and results in activation of IFN-regulatory factor 3 and the 
expression of a subset of lipopolysaccharide-inducible genes. J. Immunol. 167:5887-5894.

21. Kaisho, T., Takeuchi, O., Kawai, T., Hoshino, K., and Akira, S. 2001. Endotoxin-induced maturation of MyD88-deficient dendritic cells. J. Immunol. 166:5688-5694.

22. Yamamoto, M., et al. 2002. Cutting edge: a novel Toll/ IL-1 receptor domain-containing adapter that preferentially activates the IFN-beta promoter in the Tolllike receptor signaling. J. Immunol. 169:6668-6672.

23. Hoebe, K., et al. 2003. Identification of Lps2 as a key transducer of MyD88-independent TIR signalling. Nature. 424:743-748.

24. Yamamoto, M., et al. 2003. Role of adaptor TRIF in the MyD88-independent toll-like receptor signaling pathway. Science. 301:640-643.

25. Herrick, C.A., MacLeod, H., Glusac, E., Tigelaar, R.E., and Bottomly, K. 2000. Th2 responses induced by epicutaneous or inhalational protein exposure are differentially dependent on IL-4. J. Clin. Invest. 105:765-775.

26. Kung, T.T., et al. 1994. Characterization of a murine model of allergic pulmonary inflammation. Int. Arch. Allergy Immunol. 105:83-90.

27. Kimber, I., and Cumberbatch, M. 1992. Stimulation of Langerhans cell migration by tumor necrosis factor alpha (TNF-alpha). J. Invest. Dermatol. 99(Suppl.):48S-50S.

28. Roake, J.A., et al. 1995. Dendritic cell loss from nonlymphoid tissues after systemic administration of lipopolysaccharide, tumor necrosis factor, and interleukin 1. J. Exp. Med. 181:2237-2247.

29. Sallusto, F., Mackay, C.R., and Lanzavecchia, A. 2000. The role of chemokine receptors in primary, effector, and memory immune responses. Annu. Rev. Immunol. 18:593-620.

30. Banchereau, J., et al. 2000. Immunobiology of dendritic cells. Annu. Rev. Immunol. 18:767-811.

31. Constant, S.L., et al. 2002. Resident lung antigen-presenting cells have the capacity to promote Th2 $\mathrm{T}$ cell differentiation in situ. J. Clin. Invest. 110:1441-1448. doi:10.1172/JCI200216109.

32. Akimoto, T., et al. 1998. Abrogation of bronchial eosinophilic inflammation and airway hyperreactivity in signal transducers and activators of transcription (STAT)6-deficient mice. J. Exp. Med. 187:1537-1542.

33. Busse, W.W., and Lemanske, R.F., Jr. 2001. Asthma. N. Engl. J. Med. 344:350-362.

34. Henderson, W.R., Jr., et al. 1996. The importance of leukotrienes in airway inflammation in a mouse model of asthma. J. Exp. Med. 184:1483-1494.

35. Wills-Karp, M. 1999. Immunologic basis of antigen-induced airway hyperresponsiveness. Annu. Rev. Immunol. 17:255-281.

36. Kaisho, T., and Akira, S. 2003. Regulation of dendritic cell function through Toll-like receptors. Curr. Mol. Med. 3:373-385.

37. Hoebe, K., et al. 2003. Upregulation of costimulatory molecules induced by lipopolysaccharide and doublestranded RNA occurs by Trif-dependent and Trif- independent pathways. Nat. Immunol. 4:1223-1229.

38. Didierlaurent, A., et al. 2004. Flagellin promotes myeloid differentiation factor 88-dependent development of Th2-type response. J. Immunol. 172:6922-6930.

39. Pasare, C., and Medzhitov, R. 2003. Toll-like receptors: balancing host resistance with immune tolerance. Curr. Opin. Immunol. 15:677-682.

40. Pasare, C., and Medzhitov, R. 2003. Toll pathway-dependent blockade of CD4+CD25+ T cellmediated suppression by dendritic cells. Science. 299:1033-1036.

41. Adachi, O., et al. 1998. Targeted disruption of the MyD88 gene results in loss of IL-1- and IL-18mediated function. Immunity. 9:143-150.

42. Cohn, L., Homer, R.J., Niu, N., and Bottomly, K. 1999. T helper 1 cells and interferon gamma regulate allergic airway inflammation and mucus production. J. Exp. Med. 190:1309-1318.

43. Seymour, B.W., Gershwin, L.J., and Coffman, R.L. 1998. Aerosol-induced immunoglobulin (Ig)-E unresponsiveness to ovalbumin does not require $\mathrm{CD}^{+}$or $\mathrm{T}$ cell receptor (TCR)-gamma/delta+ $\mathrm{T}$ cells or interferon (IFN)-gamma in a murine model of allergen sensitization. J. Exp. Med. 187:721-731.

44. Lutz, M.B., et al. 1999. An advanced culture method for generating large quantities of highly pure dendritic cells from mouse bone marrow. J. Immunol. Methods. 223:77-92. 